\title{
Médiévales
}

Langues, Textes, Histoire

65 | automne 2013

Le couple dans le monde franc

\section{Innocent III. Un pape de papier}

\section{Arnaud Fossier}

\section{OpenEdition}

\section{Journals}

Édition électronique

URL : https://journals.openedition.org/medievales/7138

DOI : $10.4000 /$ medievales. 7138

ISSN : $1777-5892$

\section{Éditeur}

Presses universitaires de Vincennes

\section{Édition imprimée}

Date de publication : 1 décembre 2013

Pagination : 179-188

ISBN : 978-2-84292-396-9

ISSN : 0751-2708

\section{Référence électronique}

Arnaud Fossier, «Innocent III. Un pape de papier », Médiévales [En ligne], 65 | automne 2013, mis en ligne le 20 janvier 2014, consulté le 23 avril 2022. URL : http://journals.openedition.org/medievales/ 7138 ; DOI : https://doi.org/10.4000/medievales.7138 
Médiévales 65, automne 2013,p. 179-188

\section{Arnaud FOSSIER}

\section{INNOCENT III. UN PAPE DE PAPIER}

Qualifié de «stupeur du monde» par ses contemporains, Innocent III (1198-1216) n'a cessé d'être considéré, jusqu'à nos jours, comme l'un des souverains pontifes les plus importants du Moyen Âge, hissé au même rang que Grégoire VII, Boniface VIII ou encore Martin V. Pourfendeur de l'hérésie «cathare» dans le Midi de la France et programmateur de la quatrième croisade, il fut à l'origine de la reconquête des États de l'Église, du plus grand concile du Moyen Âge (1215) et de la restructuration de la Curie romaine. Tout a-t-il été dit, pour autant, sur ce théologien averti, se définissant comme le représentant du Christ sur terre, et sur son legs?

Le $800^{\mathrm{e}}$ anniversaire du début de son pontificat fut l'occasion, il y a une quinzaine d'années, de nouvelles publications à son sujet ${ }^{1}$, et la parution, il y a deux ans, de la traduction italienne des Gesta Innocentii prouve que l'intérêt n'a pas faibli pour l'homme qui coiffa la tiare à l'âge de trente-huit ans seulement ${ }^{2}$. Les Vies ou Gestes constituent certes un genre littéraire et hagiographique codifié qui laisse à grand-peine filtrer l'impartialité historiographique, mais la publication des actes d'un colloque international tenu en 2007 permet de restituer les évolutions que les biographies des évêques de Rome connurent tout au long du Moyen Âge ${ }^{3}$. Elle met ainsi en lumière l'originalité de la geste innocentienne.

Quant au petit livre, malheureusement dénué d'apparat critique, qu'Olivier Hanne tire de sa thèse de doctorat, portant sur le parcours et la formation de

1. À commencer par l'ouvrage collectif dirigé par J. C. Moore éd., Pope Innocent III and his World, Aldershot, 1999.

2. Gesta di Innocenzo III, éd. G. Barone et A. Paravicini-Bagliani, Rome, 2011.

3. F. Bougard et M. Sot éd., Liber, gesta, histoire. Écrire l'histoire des évêques et des papes de l'Antiquité au $\mathrm{XXI}^{e}$ siècle, Turnhout, 2009. 
Lothaire de Segni avant qu'il ne prenne le nom d'Innocent ${ }^{4}$, il rappelle à notre attention qu'au tournant des $\mathrm{XII}^{\mathrm{e}}$-XIII ${ }^{\mathrm{e}}$ siècles, la réforme de l'organisation curiale et la mise en place d'une véritable «politique par correspondance»- pour reprendre le titre d'un ouvrage collectif récent sur le rôle décisif de l'épistolarité dans la construction du pouvoir politique entre le XIV et le XVIII ${ }^{\mathrm{e}}$ siècle ${ }^{5}$ - servirent les desseins universalistes du pontife ${ }^{6}$. Au terme d'un panorama partiel de ces trois ouvrages - une source traduite, un colloque et un essai -, c'est bien l'exceptionnelle production documentaire liée à ce pontificat qui ressort et constitue sans doute une rupture majeure dans l'histoire croisée de l'écrit et des dispositifs de gouvernement en Occident.

\section{Hagiographie politique}

En 1991, l'une des meilleures spécialistes de la papauté au XIII siècle, Brenda Bolton, affirmait que les Gesta d'Innocent III avaient été sous-évalués, alors même qu'il s'agissait d'une source de premier plan pour la connaissance de la vie et de l'œuvre du pontife ${ }^{7}$. Mal connus au Moyen Âge ${ }^{8}$, les Gesta n'ont subsisté sous leur forme manuscrite qu'en peu d'exemplaires (on en compte deux français datant du XIV ${ }^{\mathrm{e}}$ siècle et un romain de la fin du XIII ${ }^{\mathrm{e}}$ ou du début du $\mathrm{XIV}^{\mathrm{e}}$ siècle), mais ils ont été édités à plusieurs reprises à partir du XVII ${ }^{\mathrm{e}}$ siècle. La plupart des historiens modernes se sont appuyés sur l'édition de Migne ${ }^{9}$, encore qu'ils se soient finalement assez peu fondés sur les Gesta pour faire l'histoire des dix premières années du pontificat d'Innocent ${ }^{10}$. Quant à l'édition plus récente de

4. O. Hanne, Lothaire de Segni (1160-1198): Formation intellectuelle et politique du pape Innocent III, soutenue en 2010 à l'Université de Provence, sous la direction de C. CAROzZI.

5. J. Boutier, S. Landi, O. Rouchon éd., La Politique par correspondance. Les usages politiques de la lettre en Italie (XIV -XVII ${ }^{e}$ siècle), Rennes, 2008. À ce sujet, pour l'ensemble de la période médiévale, on peut mentionner les deux programmes de recherche en cours, «Les correspondances en Italie ( $\mathrm{v}^{\mathrm{e}}-\mathrm{XV}^{\mathrm{e}}$ siècle) » (Università di Trieste, École française de Rome) et «L'épistolaire politique, France et monde francophone $\left(\mathrm{v}^{\mathrm{e}}-\mathrm{XV}^{\mathrm{e}}\right.$ siècle)» (Université Paris-Ouest, Université Paris-Sorbonne).

6. O. Hanne, Innocent III : la stupeur du monde, Paris, 2012.

7. B. Bolton, «Too Important to Neglect: The Gesta Innocentii PP III», Innocent III : Studies on Papal Authority and Pastoral Care, Variorum reprints, 1995 [1991].

8. K. Pennington, Pope and Bishops. The Papal Monarchy in the Twelfth and Thirteenth Centuries, Philadelphie, 1984, p. 54.

9. Gesta Innocentii (PL 214, col. 17-228).

10. Exceptons H. ElKan, Die Gesta Innocentii III im Verhältnis zu den Registen desselben Papstes, Heidelberg, 1876; Y. LefÈBvre, «Innocent III et son temps vus de Rome. Étude sur la biographie anonyme de ce pape. Résumé du Mémoire présenté à l'Académie des inscriptions», Mélanges d'archéologie et d'histoire, 61, 1949, p. 242-245 ; H. Tillmann, Papst Innocenz III, Bonn, 1954; V. PfafF, «Die Gesta Innocenz III und das Testament Heinrichs VI», Zeitschrift der Savigny- 
David R. Gress-Wright, elle est malheureusement restée confidentielle ${ }^{11}$. Assez curieusement d'ailleurs, le traducteur italien des Gesta - rappelons qu'en 2004 était parue la traduction anglaise de James Powell ${ }^{12}$ - prend comme édition de référence celle de Migne. Si les instruments de lecture que sont la bibliographie, l'index des noms de lieux et de personnages, ainsi que la liste des cardinaux cités dans les Gesta, s'avèrent précieux, la biographie d'Innocent III écrite par Werner Maleczek n'est, quant à elle, que le décalque de celle parue dans l'Enciclopedia dei papi $i^{13}$, et la courte introduction de Giulia Barone reprend en grande partie un article publié en $2001^{14}$.

L'historienne y réexplique d'abord pourquoi les Gesta n'ont pas été écrits d'une traite: une partie a été rédigée en 1203, à un moment où Innocent III était si malade que son entourage songeait à une mort imminente, mais n'a été actualisée et complétée qu'en 1208. Certainement d'origine romaine, l'auteur est un familier de la Chancellerie, doté d'une solide culture juridique, comme en témoigne sa connaissance des actes pontificaux qu'il cite ou résume abondamment au sein des Gesta. Selon G. Barone, il s'agirait de Giovanni, cardinal de Santa Maria in Cosmedin, consanguineus ou nepos du pape, attesté comme «chancelier» en 1205, et non, comme l'avait supposé J. Powell dans sa traduction des Gesta, du canoniste et compilateur des décrétales d'Innocent III, Pietro di Benevento ${ }^{15}$.

Divisés en 151 chapitres, les Gesta mettent surtout l'accent sur l'éclatante politique de récupération du Patrimoine de Saint-Pierre menée par Innocent III (chapitres 8-17), la reconquête du royaume de Sicile (chapitres 18-40), les relations diplomatiques avec les souverains européens (chapitres 41-59 et 120122), la croisade et les rapports avec l'Église grecque (chapitres 60-119). De toute évidence, l'auteur des Gesta a voulu montrer le pouvoir et l'influence du pape, y compris dans les régions les plus périphériques de la chrétienté (Arménie, Bulgarie ou Danemark), auxquelles il consacre d'amples développements. En partant ainsi de la diplomatie menée par Innocent III, il fournit les arguments

Stiftung. Kan.Abt., 1 (1964), p. 78-126, plus particulièrement p. 79-90; W. IмкAмP, Das Kirchenbild Innocenz III (1198-1216), Stuttgart, 1983, p. 10-46.

11. D. R. GRESS-WRIGHT, The Gesta Innocentii papae tertii. Text, Introduction and Commentary, Ann Arbor, 1993.

12. J. Powell, The Deeds of Pope Innocent III, by an Anonymous Author, Washington D.C., 2004.

13. W. MaleczeK, Innocenzo III, dans M. Simonetti, G. Arnaldi, M. Caravale, G. Martina éd., Enciclopedia dei papi, t. I, Rome, 2000, p. 326-350.

14. G. BARONE, «I Gesta Innocentii : politica e cultura a Roma all'inizio del Duecento », dans G. Barone, L. Capo et S. Gasparri éd., Studi sul Medioevo per Girolamo Arnaldi, Rome, 2001, p. 1-23.

15. EAD., «Introduzione», dans Gesta di Innocenzo III, p. 13-15. 
politiques en faveur de la doctrine de la plénitude de puissance ${ }^{16}$. Dans le résumé d'un mémoire de l'Académie des inscriptions et belles-lettres déjà ancien, Yves Lefèbvre notait cependant que l'auteur des Gesta n'évoquait pas la question de l'Empire, sans doute parce que celle-ci était, en 1208, loin d'être résolue ${ }^{17}$. De fait, les Gesta enregistrent le récit d'événements clos, au dénouement connu, et B. Bolton d'affirmer qu'il s'agit d' «une œuvre délibérément conçue pour célébrer les accomplissements du pape ${ }^{18} »$.

Mais s'agit-il seulement d'un panégyrique ? Et dans quelle tradition littéraire et historiographique les Gesta s'inscrivent-ils? La première tentative de rédiger une biographie des papes est celle du Liber pontificalis ${ }^{19}$, qui naît en tant que tel dans l'Italie ostrogothique de la première moitié $\mathrm{du} \mathrm{VI}^{\mathrm{e}}$ siècle - mis au point vers 535, le texte sera mis à jour entre 625 et 638 - et se substitue à la logique de la liste qui avait jusqu'alors prévalu. Il se démarque en outre du modèle biographique par excellence que sont les Vies des douze Césars de Suétone, puisque son principal objectif est de christianiser l'histoire de Rome ${ }^{20}$. Si les deux versions concurrentes du Liber prennent d'emblée une forme hagiographique, c'est aussi parce qu'elles cherchent à départager leurs champions respectifs, le pape Symmaque (498-514) et l'anti-pape Laurent (498 et 501-506) ${ }^{21}$. Constitué d'un noyau dur, le Liber se développe ensuite par ajouts successifs et s'épanouit pleinement au $\mathrm{VIII}^{\mathrm{e}}$ siècle, lorsque certaines notices biographiques deviennent de véritables «mémoires " ${ }^{22}$; selon Michel Sot, il devient même le «prototype» des gesta episcoporum, dont le genre fleurit entre le $\mathrm{VIII}^{\mathrm{e}}$ et le $\mathrm{XII}^{\mathrm{e}}$ siècle ${ }^{23}$. Pour cette seule période, on compte une vingtaine de vies d'évêques composées dans les différentes cités épiscopales d'Europe - du Nord surtout, la proximité géographique du modèle romain ayant peut-être paralysé les tentatives d'hagiographie indépendantes en Europe du Sud.

De fait, la structure des notices biographiques des papes est reprise, ou du moins adaptée, aux gestes d'évêques (ainsi d'ailleurs qu'aux biographies royales

16. D. R. Gress-Wright, The Gesta Innocentii papae tertii..., p. 110-112.

17. Y. LeFÈBVRE, «Innocent III et son temps ...», 1949, p. 242-245.

18. B. Bolton, «Too Important to Neglect...», p. 98.

19. Le Liber pontificalis, éd. L. Duchesne, 3 vol., Paris, 1886-1892 (réimpr. Paris, 1955); voir également C. Vogel, Additions et corrections. Histoire du Liber pontificalis depuis l'édition Duchesne, Paris, 1957; et, en dernière date, voir la traduction qu'en propose M. Aubrun, Le Livre des papes. Liber pontificalis (492-891), Turnhout, 2007.

20. R. MCKITTERICK, «La place du Liber pontificalis dans les genres historiographiques du haut Moyen Âge», dans F. Bougard et M. Sot éd., Liber, gesta, histoire..., p. 23-35.

21. L. DuCHESNE, «Introduction», Le Liber pontificalis, texte, introduction et commentaire, t. 1.

22. M. Sot, «Auxerre et Rome: Gesta pontificum et Liber pontificalis», dans F. BougaRd et M. Soт éd., Liber, gesta, histoire..., p. 5-20 (p. 18).

23. Ibid., p. 6. 
de la seconde moitié du XII siècle, comme celles de Frédéric Barberousse, Henri II ou Philippe Auguste ${ }^{24}$ ). Sujette à variations ${ }^{25}$, elle se laisse idéalement décomposer de la façon suivante: nom et numéro d'ordre du pape, origine géographique, durée du pontificat en année, mois et jours, cursus et récit de son élection, histoire des relations de la papauté avec le monde extérieur, décisions en matière de discipline et de liturgie, fondations et dotations d'églises, nombre d'ordinations effectuées par le pape, lieu de sépulture, date de célébration et anniversaire de sa mort. Or, les Gesta d'Innocent reprennent en partie cet agencement, puisque les sept premiers chapitres retracent le parcours de Lothaire de Segni jusqu'à son élection comme pape (8 janvier 1198), et les huit derniers recensent les fondations et dotations d'églises ainsi que les ordinations effectuées par le pape. En empruntant ainsi au cadre du Liber pontificalis, les Gesta véhiculent une représentation stable de l'institution papale.

Entre les années 870 et les années 1130, les traces d'une gestion proprement romaine de la mémoire pontificale s'étaient pourtant atténuées, puisque les notices biographiques du Liber pontificalis, quand elles ne manquaient pas, étaient redevenues fort sommaires. Dans les années 1130, le modèle du Liber pontificalis avait certes bénéficié d'un renouveau d'intérêt, lorsque le cardinal Pandolfo, «renou[ant] avec l'esprit des origines», avait continué le Liber jusqu'au pontificat d'Honorius II (1124-1130) ${ }^{26}$; mais dans la seconde moitié du XII ${ }^{\mathrm{e}}$ siècle, la voie apologétique et partisane avait finalement été privilégiée, le souci de l'impartialité étant désormais délaissé par la Curie romaine. L'entreprise romaine «affiche [alors] ses ambitions patrimoniales, sans souci de reconstituer un modèle jugé apparemment périmé ${ }^{27}$ », ce que les Gesta d'Innocent III reflètent au travers de la description enthousiaste des reconquêtes militaires successives du Patrimoine de Saint-Pierre ou de l'apologie des vertus et des divers talents du souverain pontife. Paul Payan et Guy Lobrichon vont jusqu'à affirmer, au sujet des Gesta Innocentii, mais aussi de la Vie de Grégoire IX (1227-1241) et de celle d'Innocent IV (1243-1254), que «ces œuvres [sont] rest[ées] isolées, sans terreau historiographique commun. De probable origine curiale, leur caractère officiel n'en est pas moins douteux ; la tendance hagiographique les envahit et les déleste de la sévérité traditionnelle du Liber pontificalis ${ }^{28} \gg$.

24. B. Bolton, «Too Important to Neglect...».

25. F. Bougard, «Composition, diffusion et réception des parties tardives du Liber pontificalis romain (VIII $-\mathrm{IX}^{\mathrm{e}}$ siècles)», dans F. BougarD et M. Sot éd., Liber, gesta, histoire..., p. 127-152.

26. G. Lobrichon et P. PAYAN, «Quelle écriture de l'histoire des papes d'Avignon?», dans F. Bougard et M. Sot éd., Liber, gesta, histoire..., p. 179-198 (p. 183).

27. Ibid., p. 186.

28. Ibid., p. 187. 
Cela étant, les Gesta Innocentii, auxquels le colloque consacré au genre hagiographique et historiographique des «gestes» ne fait curieusement aucune place, ne se réduisent ni au portrait emphatique ni au récit circonstancié de l'implication diplomatique d'Innocent III dans les événements politiques de son temps. Dans sa forme et sa structure mêmes, cette source dévoile surtout l'image d'un pape exceptionnellement productif sur le plan épistolaire et celle d'un génial administrateur de la chrétienté.

\section{Gouverner par lettres}

Du phénomène de bureaucratisation ou de «départementalisation» de la Curie papale, déjà bien perçu il y a quelques décennies par Jane E. Sayers ${ }^{29}$, $\mathrm{O}$. Hanne dégage rapidement les lignes de force, préférant pour sa part parler de «rationalisation» des organes de gouvernement. On pourra s'étonner que ce phénomène ait pris une ampleur sans précédent sous le pontificat d'un homme qui n'avait joué qu'un rôle mineur à la Curie durant les sept années précédant son élection, puisqu'il ne figurait guère parmi les favoris de Célestin III (1191-1198). Innocent III restructure pourtant la Chancellerie de façon décisive, en distinguant, selon O. Hanne, quatre bureaux, peuplés de notaires et de scribes placés sous les ordres d'un vice-chancelier: l'un pour la rédaction des «grosses», un autre voué à la copie de la lettre et à son enregistrement, un troisième se consacrant à l'écriture de la «minute», et un dernier composant les bulles ${ }^{30}$. Innocent III assigne un rôle majeur à la Chambre apostolique, en la chargeant de dresser l'inventaire des taxes et cens, le Liber censuum, qui justifie la réappropriation des États de l'Église. Pour finir, le système judiciaire curial se complexifie, puisque sont créées les charges de pénitencier du pape, censé recueillir les confessions des pèlerins venus à Rome et répondre à certaines des demandes d'absolution et de dispense adressées au pape, ainsi que celles des «auditeurs» de justice. Ces derniers doivent conseiller le pontife sur les litiges ecclésiastiques se présentant à la Curie; attesté en 1205-1206, l'«auditeur des lettres contredites» doit, lui, organiser la lecture des lettres de justice devant les procureurs des parties, puis expédier ces lettres après en avoir contrôlé la véracité et la légalité ${ }^{31}$.

La mise en place d'une administration professionnelle n'est évidemment pas étrangère à l'accroissement considérable de la production documentaire,

29. J. E. SAYERS, Papal Government and England during the Pontificate of Honorius III (12161227), Cambridge, 1984.

30. O. HanNe, Innocent III : la stupeur du monde..., p. 133.

31. P. HERDE, Audientia litterarum contradictarum. Untersuchungen über die päpstlichen Justizbriefe und die päpstliche Delegationsgerichtsbarkeit vom 13. bis zum Beginn des 16. Jahrhunderts, Tübingen, 1970 (Bibliothek des deutschen historischen Instituts im Rom, 31-32). 
pour ne pas dire à l'explosion épistolaire, qui marque le pontificat d'Innocent III. Une estimation fondée sur le corpus de lettres pontificales défini par Jaffé puis Potthast au $\mathrm{XIX}^{\mathrm{e}}$ siècle permet de conclure à l'augmentation de leur nombre entre 1159 et 1227, alors même qu'une partie d'entre elles seulement était enregistrée $^{32}$. J. E. Sayers compte ainsi 179 lettres enregistrées chaque année sous Alexandre III (1159-1181), 304 sous Innocent III et 239 sous Honorius III (1216-1227). Le changement n'est pas seulement quantitatif, puisque les règles et les procédures d'enregistrement sont progressivement formalisées ${ }^{33}$. Sous le pontificat d'Innocent III, en effet, une série quasi continue de registres de lettres est, pour la première fois, constituée, qui comprend environ 6000 lettres (soit 20 à $30 \%$ du total des lettres produites à la Curie $)^{34}$. Dans la notice qui précède la traduction des Gesta, W. Maleczek note à juste titre que l'objectif principal de la constitution de ces registres fut d'ordonner chronologiquement la matière première d'une nouvelle collection de décrétales.

Alors que la formation d'Innocent III en droit fut en définitive assez banale, le droit connaît, sous son règne, un développement sans précédent ${ }^{35}$. Issu de la noblesse rurale du Latium, Lothaire avait été, à l'âge de 15 ans environ, envoyé à Paris pour suivre les cours de théologie de Pierre de Corbeil, puis s'était rendu à Bologne où il avait dû rester deux ans tout au plus (entre l'automne 1187 et l'automne 1189). Les Gesta précisent qu'il s'y est formé en droit canonique ${ }^{36}$, mais la légende forgée un siècle plus tard par le décrétaliste Jean d'André, selon laquelle il aurait écouté l'enseignement d'Huguccio, le plus célèbre décrétiste de son temps, s'avère douteuse ${ }^{37}$. Si les points d'accord des deux hommes sont nombreux, concernant notamment la plénitude de puissance du pape, les preuves documentaires de leur affinité intellectuelle sont trop infimes pour qu'un rapport direct entre eux puisse être établi, d'autant, comme le rappelle O. Hanne, que

32. J. E. SAYERS, Papal Government and England...

33. R. Swanson, «Universis Christi fidelibus : the Church and its Records », dans R. BRITnELL éd., Pragmatic Literacy. East and West, 1200-1330, Woodbridge, 1997, p. 147-164; A. JAmme, «Formes et enjeux d'une mémoire de l'autorité: l'État pontifical et sa construction scripturaire aux XIII et XIV ${ }^{\mathrm{e}}$ siècles », dans L'Autorité de l'écrit au Moyen Âge (Orient-Occident), Congrès de la SHMESP, Paris, 2009, p. 341-360.

34. Die Register Innocenz III, 10 vol.,éd. O. HAGENEDER et al., Vienne, 1964-2007; pour l'année 1208-1209, voir Innocentii III Epistolae, dans PL 215, col. 1339-1612 et PL 216, col. 10-233.

35. J. E. SAYERS, Innocent III, Leader of Europe, Londres/New York, 1994, p. 94-124.

36. Gesta di Innocenzo III..., p. 53, § 2 .

37. K. Pennington, «The Legal Education of Pope Innocent III», dans Popes, Canonists and Texts, 1150-1550, Aldershot, 1993; ID., «Further Thoughts on Pope Innocent III's Knowledge of Law», dans Popes, Canonists and Texts...; C. DE Miramon, «Innocent III, Huguccio de Ferrare et Hubert de Pirovano. Droit canonique, théologie et philosophie dans les années 1180 », dans W. P. Müller et M. E. Sommar éd., Medieval Foundations of the Western Legal Tradition. A Tribute to Kenneth Pennington, Washington D.C., 2006, p. 320-346. 
leurs doctrines du mariage et de l'eucharistie diffèrent ostensiblement ${ }^{38}$. Les connaissances d'Innocent III en matière de droit canon viendraient plutôt du décrétiste Étienne de Tournai, abbé de Sainte-Geneviève auprès de qui il s'est formé à Paris, et du chancelier d'Alexandre III, Albert de Morra, canoniste et dictaministe formé à Bologne, dont il fut le protégé au milieu des années $1170^{39}$.

Si le droit eut une influence si considérable sur la correspondance d'Innocent III, c'est donc sans doute davantage parce que le style des administrateurs de la Curie, tous formés en droit canonique, en imprégnait l'écriture; des équipes de juristes entières étaient chargées de rédiger les bulles les plus importantes, telles que la Per venerabilem de 1202 affirmant la juridiction extraordinaire du souverain pontife dans les causes temporelles ${ }^{40}$. Parce qu'Innocent III cherche aussi à donner à l'institution pontificale un pouvoir qui soit supérieur à celui des autres évêques, il se préoccupe, dès les débuts de son pontificat, de la constitution d'une collection de lettres dont la portée normative soit universelle. Commencée en 1203 par Pietro Collivaccino di Benevento, notaire et chapelain d'Innocent III, la Compilatio Tertia - les deux collections précédentes n'avaient pas été reconnues officiellement - contient ainsi cinq cents lettres triées à partir des registres et voit le jour en 1209 pour, l'année suivante, être envoyée à Bologne et y être enseignée à la nouvelle génération de canonistes.

\section{La mémoire dans la peau}

Les Gesta Innocentii sont eux-mêmes truffés de textes extraits des registres de lettres du grand pape, ce qui constitue une relative nouveauté puisque, entre Étienne V (885-891) et Léon IX (1049-1054), les lettres pontificales, qui en constituaient encore la matière première aux $\mathrm{VIII}^{\mathrm{e}}-\mathrm{IX}^{\mathrm{e}}$ siècles, avaient été mises à l'écart du Liber pontificalis ${ }^{41}$. L'auteur des Gesta cite parfois intégralement les lettres d'Innocent III : dans le récit qu'il fait de la croisade de 1204, il restitue le rôle joué par le pape en prenant soin de retranscrire les lettres que le pontife a adressées à l'empereur byzantin, au patriarche de Constantinople ou encore au roi des Bulgares. Exceptionnellement, il insère des documents qui n'ont pas été conservés par ailleurs, tel le testament de l'empereur Henri VI ${ }^{42}$. La norme reste néanmoins le choix de citer les lettres contenues dans les registres.

38. O. Hanne, Innocent III..., p. 32-33.

39. Sur l'utilisation des sources du droit civil dans les lettres d'Innocent III, voir J. M. RAINER, «Innocenz III und das römische Recht», Römische historische Mitteilungen, 25 (1983), p. 15-33.

40. $X, 4,17,13$ (Fr. II, 714-716).

41. K. Herbers, «Agir et écrire: les actes des papes du IX ${ }^{\mathrm{e}}$ siècle et le Liber pontificalis », dans F. Bougard et M. Sot éd., Liber, gesta, histoire..., p. 109-126. F. Bougard, «Composition, diffusion et réception des parties tardives du Liber pontificalis romain ( $\mathrm{VIII}^{\mathrm{e}}-\mathrm{IX}^{\mathrm{e}}$ siècles)... », p. 129.

42. Gesta di Innocenzo III..., p. 76-77. 
À s'en tenir là, on conclurait à la fonction illustrative et narrative des lettres insérées dans les Gesta ou à la reprise d'une forme historiographique consistant à étayer le récit par la citation de documents authentiques. Il n'est de fait pas inconcevable que l'auteur des Gesta ait cherché à conserver la mémoire vive des œuvres du pontife, tout en la cadrant par une sorte de rigueur des faits enfermée dans chacune des lettres citées. Mais cette vision somme toute «positiviste» du document ne correspond pas à la valeur et à la fonction que les hommes de l'époque assignaient aux lettres pontificales. Au même titre que les registres de lettres, les Gesta ont surtout fait office de «conservatoire» des lettres et ont vraisemblablement servi de passerelle textuelle entre les registres et la collection de décrétales en germe dès 1203. Comme le suggère G. Barone, ce n'est sans doute pas un hasard si, cinq ans plus tard, l'écriture des Gesta cesse au moment où la Compilatio Tertia se trouve achevée...

Telle une poupée russe, la mémoire vive que portent les Gesta - celle des faits du pontife - en recèle donc une autre: une mémoire des lettres, dont la portée normative obsédait Innocent III, ayant trouvé là le moyen par excellence d'assurer, à l'échelle de la chrétienté, sa plénitude de puissance. Celle-ci ne seraitelle pas restée pure abstraction si elle ne s'était appuyée sur une administration aussi productive et organisée?

Pourtant, alors même qu'il prétendait servir le pouvoir sans frontières dont les successeurs d'Innocent III se targuèrent, ce modèle des Gesta comme conservatoire des documents authentiques fit long feu, du moins à la Curie romaine, si l'on en croit P. Payan et G. Lobrichon. L'affirmation de deux historiens n'étant guère étayée, on peut tout de même s'interroger sur la fortune des Gesta. Si l'on connaît, aujourd'hui, l'influence que le Liber pontificalis eut sur la composition des Gesta episcoporum, au travers, par exemple, du «Livre pontifical de l'église de Ravenne» composé dans les années $830-840^{43}$, on sait peut-être moins dans quelle mesure les Gesta Innocentii ont constitué une source d'influence, en matière de procédés narratifs et historiographiques, pour les biographies ultérieures, qu'elles soient d'évêques ou de princes.

Sans doute parce qu'il suit un plan presque entièrement chronologique, le livre d'O. Hanne ne fait pas véritablement émerger de l'océan des événements qui rythment le tournant des $\mathrm{XII}^{\mathrm{e}}$-XIII ${ }^{\mathrm{e}}$ siècles la question cruciale de la mémoire du pouvoir. En revanche, la traduction des Gesta, lue à la lumière du récent colloque sur l'histoire de ce genre, démontre non seulement que l'hagiographie fit office d'instrument politique, mais aussi que l'un des pontifes les plus marquants de son époque fut acteur et témoin de la «révolution» épistolaire, archivistique

43. D. M. Deliyannis, «The Liber pontificalis of the Church of Ravenna: its Relation with its Roman Model», dans F. Bougard et M. Sot éd., Liber, gesta, histoire..., p. 283-298. 
A. FOSSIER

et juridique qui contamina, au cours d'un long XIII siècle, tous les appareils de gouvernement en Occident.

Arnaud Fossier - Université de Bourgogne, Esplanade Erasme, 21000 Dijon - UMR 6298 ARTeHIS 